\title{
Development of Two Steradian EDX System for the HD-2700 FE-STEM Equipped with Dual X-Max 100 TLE Large Area Windowless SDDs
}

Takahito Hashimoto $^{1}$, Keiji Tamura ${ }^{1}$, Hiromi Inada ${ }^{1}$, Keitaro Watanabe ${ }^{1}$, Yoshihiro Ohtsu ${ }^{1}$, Yuya Suzuki ${ }^{1}$, Takahiro Sato ${ }^{1}$, Takashi Kanemura ${ }^{1}$, Simon Burgess ${ }^{2}$, James Holland ${ }^{2}$, Iain Anderson ${ }^{2}$, Susumu Yamaguchi $^{3}$, and Kuniyasu Nakamura ${ }^{1}$

1. Science \& Medical System Business Group, Hitachi High-Technologies Corporation, 882 Ichige, Hitachinaka, Ibaraki 312-8504, Japan.

2. Nano Analysis, Oxford Instruments, High Wycombe Bucks, HP12 3SE, UK.

3. Nano Analysis, Oxford Instruments K.K., Shinagawa, Tokyo 140-0002, Japan.

The model HD-2700 [1] $200 \mathrm{kV}$ spherical aberration (Cs) corrected dedicated Scanning Transmission Electron Microscope (STEM) has been used for analyzing nano- to subnano-area targets in the fields of nanoscience and nanotechnology with Energy Dispersive X-ray spectrometry (EDX). The Cs corrector [2] enables the formation of sub-nanometer probe size with several hundred to a thousand pico amperes of probe current, but still EDX detectors with much higher sensitivity are desired. Recent adaptation of Silicon Drift Detector (SDD) technology [3] accelerated the counting rate of detection and enhancement of detector active area. These features are suitable to improve analytical sensitivity. Using a windowless high solid angle SDD, high sensitivity elemental analysis can be achieved [4].

In this study, Oxford Instruments X-Max ${ }^{\mathrm{N}}$ 100TLE SDD have been mounted to the HD-2700. This SDD exploits a new $100 \mathrm{~mm}^{2}$ sensor and a windowless configuration. These features are suitable to make highly sensitive TEM/STEM/EDX system especially for light elements. Due to the shape of the SDD sensor and geometry of pole piece area, detection solid angle of over 1 sr with take-off angle of 20 degrees or higher has been achieved. Higher take-off angle is also important for the system to obtain X-ray data with higher peak to background ratio, because of different angular distributions of characteristic and continuous $\mathrm{X}$ rays [5]. The objective lens of the HD-2700 was modified to accommodate this detector. In response to the demand of further increment for sensitivity, objective lens modification enables a second detector to be mounted at the opposite side to the first one, with azimuth angle of 90 and 270 degrees to the specimen-tilt axis. Figure 1 shows the dual detector layout. Table 1 shows specifications of the X-Max ${ }^{N}$ 100TLE for the HD-2700. With both standard (STD) and high resolution (HR) type pole pieces, over 1 sr of detection solid angle is achieved. Dual configuration doubles the nominal solid angle.

Figure 2 shows a comparison of specimen tilt dependency of Ni-K $\alpha$ line intensity acquired from a 65 $\mathrm{nm}$ thick Nickel Oxide specimen with a single detector and dual detectors. An HD-2700 with HR lens was used. The intensity of Ni-K $\alpha$ line acquired with a single detector is increased from an input rate of 15 kilo counts per second ( $\mathrm{kcps}$ ) to $22 \mathrm{kcps}$ as the specimen tilted from 0 degrees to 20 degrees, because absorption in the specimen and shading of specimen holder are reduced. Therefore, the higher tilt angle ensures improved acquisition efficiency as shown in Figure 2 (a). Some specimens, such as semiconductor devices, interfaces, or those that require crystallographic orientation may not always be analyzed with optimum tilt condition. Figure 2 (b) shows Ni-K $\alpha$ spectra acquired with the dual detector setup from tilt angles of 0 degrees to -15 degrees. Negative tilt angles are disadvantageous for a single detector due to holder shading, but total input rates were constantly about $34 \mathrm{kcps}$ in this tilt range, and the intensity of the $\mathrm{Ni}-\mathrm{Ka}$ line at various tilt angles does not reveal big changes in contrast to the results 
shown by a single detector. In this geometry, tilt setting which is disadvantageous for one detector is compensated by the advantageous tilt condition of the other detector. Application data of the HD-2700 with Dual X-Max ${ }^{\mathrm{N}} 100 \mathrm{TLE}$ system shall be introduced.

\section{References:}

[1] H. Inada, et al, J. Electron Microsc., 58 (2009), p.111-122.

[2] M. Haider, H. Rose, S. Uhlemann, B. Kabius and K. Urban, J. Elec. Microsc., 47 (1998), p. 395-405.

[3] E. Gatti, P. Rehak, Nucl. Instr. and Meth. A 225 (1984), p. 608-614.

[4] K. Tamura, et al, Microsc. Microanal. 19 (Suppl 2) (2013), p. 1192-1193.

[5] C.E. Fiori, C.R. Swyt, and J.R. Ellis, in "PRINCIPLES OF ANALYTICAL ELECTRON MICROSCOPY”, ed. D.C.Joy et al., (PLENUM, New York), p. 420.

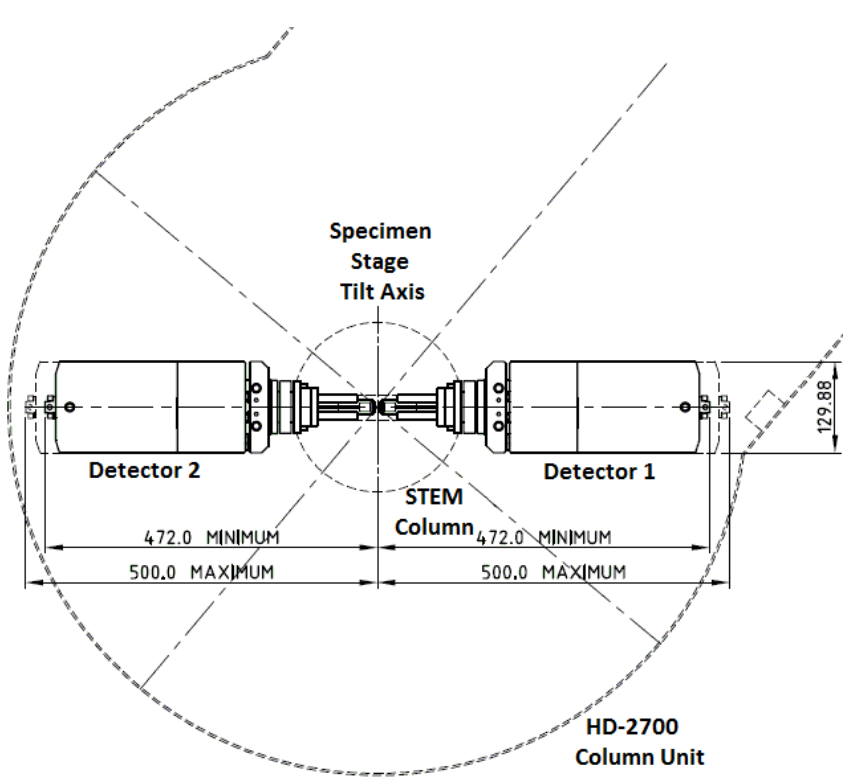

Table 1. Specifications of the $\mathrm{X}-\mathrm{Max}^{\mathrm{N}}$ 100TLE Detector for the HD-2700 STEMs

\begin{tabular}{|c|c|c|}
\hline Objective Lens & \multicolumn{1}{|c|}{ STD } & \multicolumn{1}{c|}{ HR } \\
\hline Solid Angle & $1.07 \mathrm{sr}$ & $1.04 \mathrm{sr}$ \\
Single (Dual) & $(2.15 \mathrm{sr})$ & $(2.07 \mathrm{sr})$ \\
\hline Take-Off Angle & $24.8 \mathrm{deg}$. & $22.8 \mathrm{deg}$. \\
\hline FWHM & & \\
@ Mn-K $\alpha$ & $127 \mathrm{eV}$ & $127 \mathrm{eV}$ \\
\hline
\end{tabular}

Figure 1. Dual X-Max ${ }^{\mathrm{N}}$ 100TLEs Layout in the HD-2700 Column Unit. Detector 1 is mounted at the standard EDX port. The additional Detector 2 uses the IP-3 port. Both ports are perpendicular to the specimen stage tilt axis.
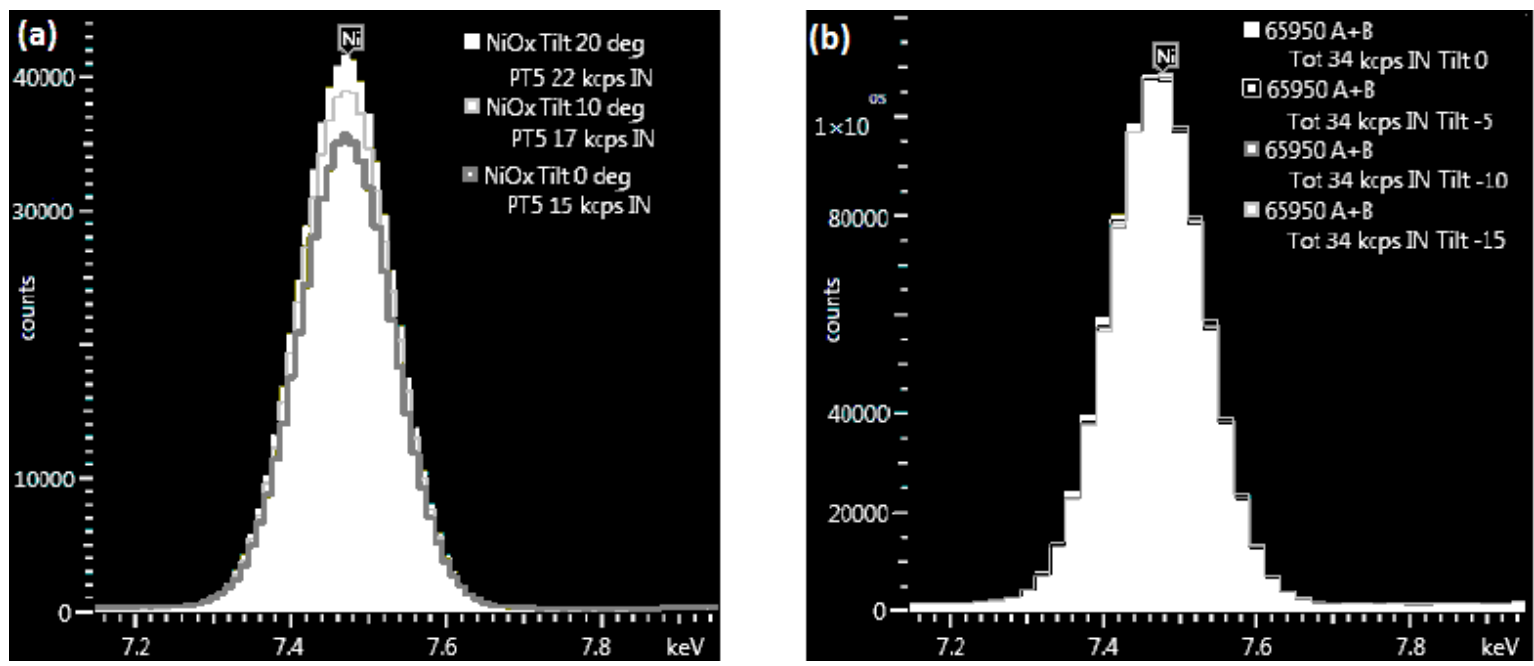

Figure 2. Comparison of specimen tilt angle dependency of $\mathrm{Ni}-\mathrm{K} \alpha$ line intensity from a Nickel Oxide thin film specimen acquired with (a) single detector (Detector 1 only) and (b) dual detectors 